\title{
LINEAR MAPS DO NOT PRESERVE COUNTABLE-DIMENSIONALITY
}

\author{
MLADEN BESTVINA AND JERZY MOGILSKI
}

\begin{abstract}
Examples of linear maps between normed spaces are constructed, including a one-to-one map from a countable-dimensional linear subspace of $I_{2}$ onto $I_{2}$. We prove that the linear span of a countable-dimensional linearly independent subset of a normed linear space is, in many cases, countable dimensional.
\end{abstract}

1. Introduction. In this note we shall prove that for a given separable Banach space $Y$ there exists a one-to-one, continuous linear surjection $F: E \rightarrow Y$, where $E$ is a normed linear space, which is a countable union of zero-dimensional sets. The space $E$ will be obtained as the linear span of a carefully embedded zero-dimensional metric space into a Banach space. If $Y$ is a Hilbert space then $E$ can be chosen to be a linear subspace of the Hilbert space.

In the proof we use the well-known construction of an embedding of a metric space onto a linearly independent subset of a Banach or Hilbert space briefly described in $\$ 2$. In $\$ 3$ we will prove that the linear span of a carefully embedded countable-dimensional, separable metric space is also countable dimensional. In $\S 4$ we will construct some examples of linear maps "raising" topological dimension.

2. The standard embedding into $l_{p}$-spaces, $1 \leqslant p \leqslant \infty$. Recall that for a set $S$ we can define normed spaces $l_{p}(S), 1 \leqslant p \leqslant \infty$. For $1 \leqslant p<\infty, l_{p}(S)$ consists of functions $z: S \rightarrow \mathbf{R}$ such that $\sum_{s \in S}|z(s)|^{p}<\infty$ with usual addition and scalar multiplication. The $p$-norm of $z \in l_{p}(S)$ is $\|z\|_{p}=\left(\sum_{s \in S}|z(s)|^{p}\right)^{1 / p}$.

The space $l_{\infty}(S)$ consists of all bounded functions $z: S \rightarrow \mathbf{R}$. The $\infty$-norm of $z \in l_{\infty}(S)$ is $\|z\|_{\infty}=\sup _{s \in S}|z(s)|$.

Let $X$ be a metric space with metric $d$ bounded by 1 . In this section we briefly describe an embedding $h: X \rightarrow l_{p}(S), 1 \leqslant p \leqslant \infty$, with certain nice properties.

First consider the case $1 \leqslant p<\infty$. The construction for $p=2$ can be found in [BP, p. 193].

For $n=1,2, \ldots$ fix a locally finite partition of unity $\{\phi\}_{\phi \in \Lambda_{n}}$ such that $d(x, y) \geqslant$ $1 / 2^{n}$ implies $\phi(x) \cdot \phi(y)=0$ for all $\phi \in \Lambda_{n}$. Then for $x \in X$ define $\hat{x}: \Lambda \rightarrow \mathbf{R}$,

Received by the editors April 10, 1984. Partially presented at a Special Session of the 810th meeting of the AMS at the University of Notre Dame, April 6-7, 1984.

1980 Mathematics Subject Classification. Primary 46B99, 54F45; Secondary 54C25, 55P10.

Key words and phrases. Countable dimension, normed linear spaces, continuous linear maps, Hamel basis, linear span, embeddings onto linearly independent sets. 
where $\Lambda=\bigcup_{n=1}^{\infty} \Lambda_{n}$, by $\hat{x}(\phi)=\left[1 / 2^{n} \cdot \phi(x)\right]^{1 / p}$, for $\phi \in \Lambda_{n}$. Then

$$
\hat{x} \in l_{p}(\Lambda) \text { and }\|\hat{x}\|_{p}=\left(\sum_{n=1}^{\infty} \frac{1}{2^{n}}\left(\sum_{\phi \in \Lambda_{n}} \phi(x)\right)\right)^{1 / p}=1 .
$$

2.1. Proposition. The function $h: X \rightarrow l_{p}(\Lambda)$ given by $h(x)=\hat{x}$ has the following properties.

(1) $h$ is an embedding,

(2) $h(X)$ is a linearly independent subset, and

(3) for every $x \in X$, and every closed subset $F \subseteq X$ with $x \notin F$, there exists a continuous linear functional $\psi: l_{p}(\Lambda) \rightarrow \mathbf{R}$ such that $\psi(h(F))=\{0\}$ and $\psi(h(x)) \neq 0$.

For the proof of (1) and (2) (for the case $p=2$ ), see [BP, p. 193]. Property (3) is built into the construction, since we can use the "projection onto a $\phi$-coordinate", i.e. the functional $\psi(z)=z(\phi)$ for appropriately chosen $\phi \in \Lambda_{n}$. (Pick $n \geqslant 1$ such that $1 / 2^{n}<d(x, F)$, and find $\phi \in \Lambda_{n}$ such that $\phi(x) \neq 0$.)

Note that if $X$ is a separable metric space, we can arrange that $\Lambda$ is countable.

The construction for $p=\infty$ can also be found in [BP, p. 49]. Define the space $Y=X \cup\left\{y_{0}\right\}$, with the metric $\tilde{d}$ that extends $d$ and has the property that $\tilde{d}\left(x, y_{0}\right)$ $=1$ for $x \in X$. Let $A=\left\{\alpha: Y \rightarrow \mathbf{R} ; \alpha\left(y_{0}\right)=0,\left|\alpha\left(y_{1}\right)-\alpha\left(y_{2}\right)\right| \leqslant \tilde{d}\left(y_{1}, y_{2}\right)\right.$ for all $\left.y_{1}, y_{2} \in Y\right\}$. Finally, define $h: X \rightarrow l_{\infty}(A)$ by $h(x)=\hat{x}$, where $\hat{x}(\alpha)=\alpha(x)$.

2.2 Proposition. The map $h$ is an isometry, $h(X)$ is a linearly independent subset of $l_{\infty}(A)$, and

(4) for every $x \in X$ and every closed subset $F \subseteq X$ with $x \notin F$ there exists $a$ continuous linear functional $\psi: l_{\infty}(A) \rightarrow \mathbf{R}$ such that $\psi(h(F))=\{0\}$ and $\psi(h(x)) \neq 0$.

Again, (4) can be proved using the appropriate projection. If we set $\alpha(y)=$ $\tilde{d}\left(y, F \cup\left\{y_{0}\right\}\right)$, then the functional $\psi: l_{\infty}(A) \rightarrow \mathbf{R}$ defined by $\psi(z)=z(\alpha)$, has the desired property. The rest is proved in [BP].

3. Countable-dimensional linear spaces. We can construct many interesting normed spaces by taking span $h(X)$ where $h: X \rightarrow E$ is an embedding of a metric space into a normed space such that $h(X)$ is a linearly independent subset (e.g. we can use the construction described in $\S 2$ ). The question we want to address in this section is: When is span $h(X)$ countable dimensional? (A separable metric space $Z$ is countable dimensional if it can be represented as a countable union of zero-dimensional subsets.) The obvious necessary condition is that $X$ must be countable dimensional.

3.1. Example. Choose a Hamel basis $X$ of $l_{2}=l_{2}(\mathbf{N})$, and let $f: C \rightarrow X$ be a one-to-one surjective map from a zero-dimensional separable metric space $C$. Assuming that $C \subseteq\left[\frac{1}{2}, 1\right]$, we set

$$
X^{\prime}=\left\{\frac{f^{-1}(x)}{\|x\|_{2}} \cdot x: x \in X\right\} .
$$

Then $X^{\prime}$ is also a Hamel basis for $l_{2}$, and $x^{\prime} \mapsto\left\|x^{\prime}\right\|_{2}$ defines a homeomorphism $X^{\prime} \approx C$. Therefore $\operatorname{dim} X^{\prime}=0$ and span $X^{\prime}=l_{2}$ (which is not countable dimensional). 
It is known (cf. [BP, p. 282]) that if $X$ is a countable union of finite-dimensional compacta, then span $h(X)$ is countable dimensional (for every embedding $h: X \rightarrow E$ such that $h(X)$ is a linearly independent subset of $E)$. We prove in this section that if $h$ is a "nice" embedding, then span $h(X)$ is countable dimensional, provided $X$ is countable dimensional. The standard embeddings described in $§ 2$ possess this nice property.

3.2. TheOREM. Let $h: X \rightarrow E$ be an embedding of a countable dimensional separable metric space $X$ into a linear metric space $E$ such that $h(X)$ is a linearly independent subset of $E$. Suppose that $h(X)$ satisfies the following property.

For every $x \in X$ and every closed subset $F \subseteq X$ with $x \notin F$ there exists a continuous linear functional $\psi: E \rightarrow \mathbf{R}$ such that $\psi(h(F))=\{0\}$ but $\psi(h(x)) \neq 0$.

Then span $h(X) \subseteq E$ is countable dimensional.

Proof. To an ordered collection $\left(N ; i_{1}, \ldots, i_{s}\right)$ of positive integers with $i_{1}<\ldots$ $<i_{s}$ we assign the collection $T\left(N ; i_{1}, \ldots, i_{s}\right)=\left\{\left(t_{1}, \ldots, t_{m}\right) \in \mathbf{R}^{m} ;-N \leqslant t_{1}=\ldots\right.$ $=t_{i_{1}}, t_{i_{1}}+1 / N \leqslant t_{i_{1}+1}=\cdots=t_{i_{2}}, t_{i_{2}}+1 / N \leqslant t_{i_{2}+1}=\cdots=t_{i_{3}}, \ldots, t_{i_{s-1}}+1 / N$ $\leqslant t_{i_{s-1}+1}=\cdots=t_{i_{s}}=t_{m} \leqslant N,\left|t_{i}\right| \geqslant 1 / N$ for all $\left.i\right\}$. Denote by $X\left(N ; i_{1}, \ldots, i_{s}\right)$ the collection of points $z$ in span $h(X)$ that can be represented as $z=t_{1} h\left(x_{1}\right)+\cdots+$ $t_{m} h\left(x_{m}\right)$ for some $\left(t_{1}, \ldots, t_{m}\right) \in T\left(N ; i_{1}, \ldots, i_{s}\right)$, and some $\left(x_{1}, \ldots, x_{m}\right) \in X^{m}$ with $x_{i} \neq x_{j}$ for $i \neq j$. Note that $\operatorname{span} h(X)-\{0\}$ can be represented as the countable union of such subsets (for different choices of $\left(N ; i_{1}, \ldots, i_{s}\right)$ ). Consequently, it suffices to prove that $X\left(N ; i_{1}, \ldots, i_{s}\right)$ is countable dimensional.

Define a map $\chi:\left\{\left(x_{1}, \ldots, x_{m}\right) \in X^{m}: x_{i} \neq x_{j}\right.$ for $\left.i \neq j\right\} \times T\left(N ; i_{1}, \ldots, i_{s}\right) \rightarrow$ $X\left(N ; i_{1}, \ldots, i_{s}\right)$ by

$$
\chi\left(x_{1}, \ldots, x_{m}, t_{1}, \ldots, t_{m}\right)=t_{1} h\left(x_{1}\right)+\cdots+t_{m} h\left(x_{m}\right) .
$$

Noting that the domain of $\chi$ is countable dimensional (since it is contained in $\left.[-N, N]^{m} \times X^{m}\right)$, the rest of the proof follows from the next two lemmas.

3.3. LEMMA. $\chi$ is a closed $i_{1} !\left(i_{2}-i_{1}\right)$ ! $\cdots\left(i_{s}-i_{s-1}\right)$ !-to-1 surjection.

3.4. LemMA. If $f: X \rightarrow Y$ is a closed q-to-1 map between separable metric spaces $(q \geqslant 1)$, and if $X$ is countable dimensional, then $Y$ is countable dimensional.

Proof of Lemma 3.3. From the uniqueness of the representation of $z \in$ span $h(X)-\{0\}$ as a linear combination of elements in $h(X)$ (up to a permutation), it follows that $\chi$ is a $i_{1} !\left(i_{2}-i_{1}\right)$ ! $\cdots\left(i_{s}-i_{s-1}\right)$ !-to-1 surjection. To show that $\chi$ is closed, it suffices to prove that if $\left(z_{k}\right)_{k=1}^{\infty}$ is a sequence in the domain of $\chi$, and if $\chi\left(z_{k}\right) \rightarrow \chi(z)$ for some $z$ in the domain of $\chi$, then $\left(z_{k}\right)_{k=1}^{\infty}$ has a convergent subsequence. To set the notation, let $z_{k}=\left(x_{1}^{k}, \ldots, x_{m}^{k}, t_{1}^{k}, \ldots, t_{m}^{k}\right), \quad z=$ $\left(x_{1}, \ldots, x_{m}, t_{1}, \ldots, t_{m}\right)$. Passing to a subsequence, we may assume that

(5) $t_{i}^{k} \rightarrow t_{i}^{0}, i=1, \ldots, m$, and

(6) $\left(x_{i}^{k}\right)_{k=1}^{\infty}$ either converges, or does not have a convergent subsequence, $i=$ $1, \ldots, m$. 
For $i=1, \ldots, m$ let $\Omega_{i}=\left\{j: x_{j}^{k} \rightarrow x_{i}\right\}$. By (*) we can choose a linear functional $\psi: E \rightarrow \mathbf{R}$ such that

(7) $\psi\left(h\left(x_{i}\right)\right) \neq 0$,

(8) $\psi\left(h\left(x_{j}\right)\right)=0$, for $j \neq i$, and

(9) $\psi\left(h\left(x_{j}^{k}\right)\right)=0$, for all $j \notin \Omega_{i}$ and all but finitely many values of $k$.

Passing to the limit of the left-hand side in $\psi \chi\left(z_{k}\right) \rightarrow \psi \chi(z)$ it follows that

$$
\sum_{j \in \Omega_{i}} t_{j}^{0}=t_{i}
$$

Since $t_{i} \neq 0$, we must have $\Omega_{i} \neq \phi(i=1, \ldots, m)$. Moreover, since $\Omega_{1}, \ldots, \Omega_{m}$ are pairwise disjoint subsets of $\{1, \ldots, m\}$, it follows that card $\Omega_{i}=1, i=1, \ldots, m$, and hence $\Omega_{1} \cup \cdots \cup \Omega_{m}=\{1, \ldots, m\}$. In particular, $\left(z_{k}\right)_{k=1}^{\infty}$ converges.

Proof of Lemma 3.4. For $p=1,2, \ldots$ denote $Y_{p}=\left\{y \in Y: d\left(x, x^{\prime}\right) \geqslant 1 / p\right.$ for all $x, x^{\prime} \in f^{-1}(y)$ with $\left.x \neq x^{\prime}\right\}$. Since $Y=Y_{1} \cup Y_{2} \cup \cdots$ it suffices to show that $Y_{p}$ is countable dimensional for each $p$. We will prove that $f \mid f^{-1}\left(Y_{p}\right): f^{-1}\left(Y_{p}\right) \rightarrow Y_{p}$ is a local homeomorphism (and hence, by separability, $Y_{p}$ can be covered by countably many open sets, each of which embeds into $\left.f^{-1}\left(Y_{p}\right) \subseteq X\right)$. For $x \in f^{-1}\left(Y_{p}\right)$ consider

$$
f \mid: \overline{N_{1 / 3 p}(x)} \cup f^{-1}\left(Y_{p}\right) \rightarrow f \overline{\left(N_{1 / 3 p}(x)\right)} \cap Y_{p}
$$

$\overline{\left(N_{1 / 3 p}(x)\right.}$ is the closed $1 / 3 p$-ball about $\left.x\right)$. Clearly, this a closed one-to-one surjection. To finish the argument, observe that $\overline{f\left(N_{1 / 3 p}(x)\right)} \cap Y_{p}$ contains a neighborhood of $f(x)$ in $Y_{p}$. (If $y \in Y_{p}$ is close enough to $f(x)$, then $f^{-1}(y)$ is contained in $1 / 3 p$-neighborhood of $f^{-1} f(x)$. Since $y \in Y_{p}$, no pair of points of $f^{-1}(y)$ can be contained in $1 / 3 p$-neighborhood of some $x^{\prime} \in f^{-1} f(x)$. Using that card $f^{-1}(y)=$ card $f^{-1} f(x)$, it follows that $f^{-1}(y)$ intersects $\overline{N_{1 / 3 p}(x)}$.)

4. Examples of linear maps raising topological dimension. We will need the following observation concerning linear extension of continuous maps.

4.1 Lemma. Let $X$ be a Hamel basis in a normed linear space $\left(E,||_{1}\right)$ and let $F$ : $E \rightarrow Y$ be a linear map of $E$ into a Banach space $\left(Y, \|_{2}\right)$ given by

$$
\begin{gathered}
F\left(\sum_{i=1}^{n} t_{i} x_{i}\right)=\sum_{i=1}^{n} t_{i} f\left(x_{i}\right), \quad \text { where } x_{i} \in X, t_{i} \text { is a real number for } i=1, \ldots, n, \\
\text { and } f: X \rightarrow Y \text { is a continuous map. }
\end{gathered}
$$

Let || be a new norm on $E$ defined by $|x|=\left(|x|_{1}^{2}+|F(x)|_{2}^{2}\right)^{1 / 2}$. Then:

(i) the map $F:(E,||) \rightarrow\left(Y, \|_{2}\right)$ is continuous,

(ii) if the Hamel basis $X$ satisfies the condition (*) of 3.2 with respect to the norm $\mid \|_{1}$, then $X$ satisfies $(*)$ with respect to $\|$,

(iii) the norm || induces the same topology on $X$.

Let us observe that $F$ may not be continuous as a map of $\left(E, \mid \|_{1}\right)$ into $\left(Y,||_{2}\right)$. For instance, let $E=\operatorname{span} X$, where $X=\left\{\left(x_{i}\right) \in l_{2}: x_{i}=t^{i}, t \in\left[\frac{1}{3}, \frac{2}{3}\right]\right\}$. Let $f$ be a continuous real-valued function on $X$ such that $f^{-1}(0)=\left\{\left(t^{i}\right) \in X: t \in\left[\frac{1}{3}, \frac{1}{2}\right]\right\}$ and $f^{-1}(1)=\left(\left(\frac{2}{3}\right)^{i}\right)$. The set $X$ is a Hamel basis for $E$ and span $f^{-1}(0)$ is dense in $E$ (cf. [BP, p. 267]). Hence the linear extension $F$ of $f$ is not continuous because $F(x)=0$ for $x \in \operatorname{span} f^{-1}(0)$ and $F\left(\left(\left(\frac{2}{3}\right)^{i}\right)\right)=1$. 
Let us recall that a linear subspace of the Hilbert space is called a pre-Hilbert space.

4.2. ExAmple. There exists a continuous one-to-one linear surjection $F: E \rightarrow l_{2}$ of a countable-dimensional pre-Hilbert space $E$ onto $l_{2}$.

Proof. Let $X$ be the zero-dimensional Hamel basis of the Hilbert space $l_{2}$ constructed in $\S 3$, and let $h: X \rightarrow l_{2}$ be an embedding of $X$ onto linearly independent subset of $l_{2}$ described in $\S 2$. Let us consider the linear space $E=\operatorname{span} h(X)$ with the norm given by $|y|=\left(\|y\|_{2}^{2}+\|F(y)\|_{2}^{2}\right)^{1 / 2}$ for $y \in E$, where $F: E \rightarrow l_{2}$ is the linear extension of the map $h^{-1}: h(X) \rightarrow X$. (Note that the norm $\|$ is induced by an inner product $x * y=x \cdot y+F(x) \cdot F(y)$ for $x, y \in E$. Thus the linear completion of $E$ is isomorphic to $l_{2}$, and hence $E$ is a pre-Hilbert space.)

By Lemma 4.1 and Theorem $3.2,(E,||)$ is a countable-dimensional pre-Hilbert space, and the linear map $F:(E, \mid) \rightarrow\left(l_{2},\|\|_{2}\right)$ is a continuous, one-to-one surjection.

Repeating the above construction we obtain

4.3. Example. Let $Y$ be a separable Banach space. There exists a continuous, one-to-one linear surjection $F: E \rightarrow Y$ of a countable-dimensional normed linear space $E$ onto $Y$.

A metric space $X$ is $\sigma$-finite-dimensional-compact if $X$ is a countable union of finite-dimensional compacta. The next example answers a question posed in [MM].

4.4 ExAmple. There exists a continuous linear surjection $F$ of a $\sigma$-finite-dimensional-compact pre-Hilbert space $V$ onto the pre-Hilbert space $\Sigma=\left\{\left(t_{i}\right) \in l_{2}\right.$ : $\left.\sum_{i=1}^{\infty}\left(i t_{i}\right)^{2}<\infty\right\}$ which contains the infinite-dimensional compact convex set $Q=$ $\left\{\left(t_{i}\right) \in l_{2}: \sum_{i=1}^{\infty}\left(i t_{i}\right)^{2} \leqslant 1\right\}$.

Proof. Let $f: I \rightarrow Q$ be a continuous surjection of the interval $I=[0,1]$ onto $Q$ and let $h: I \rightarrow l_{2}$ be an embedding onto a linearly independent subset of $l_{2}$ described in $\S 2$. The linear extension $F: \operatorname{span} h(I) \rightarrow \sum$ of the map $f h^{-1}: h(I) \rightarrow Q$ is a continuous linear surjection of the $\sigma$-finite-dimensional-compact pre-Hilbert space $V=(\operatorname{span} h(I),||)$ onto $\sum$, where $|x|=\left(\|x\|_{2}^{2}+\|F(x)\|_{2}^{2}\right)^{1 / 2}$ (see [BP, p. 282] for the proof that span $h(I)$ is $\sigma$-finite-dimensional-compact).

4.5. EXAmple (CF. [MM]). There exists an open linear surjection of a $\sigma$-finitedimensional-compact pre-Hilbert space onto a pre-Hilbert space which is not countable dimensional.

Proof. Let $F: V \rightarrow \sum$ be the map constructed in Example 4.4. Let $Y=\sigma / \operatorname{Ker} F$ be the quotient space. Then the quotient map $T: V \rightarrow Y$ is open. The space $Y$ cannot be countable dimensional because it is $\sigma$-compact and we can map $Y$ onto $\sum$ by a continuous, one-to-one map.

4.6. REMARK. Each continuous linear map between metric linear spaces is a $U V^{\infty}$-map (a map $f: X \rightarrow Y$ is a $U V^{\infty}$-map if for every $y \in Y$ and every open set $U$ containing $y$, there exists an open set $V, y \in V \subset U$, such that $f^{-1}(V)$ is contractible in $\left.f^{-1}(U)\right)$. By $[\mathbf{H}]$ the linear maps constructed in $\$ 4$ are fine homotopy equivalences (the map $f: X \rightarrow Y$ is a fine homotopy equivalence if for every open cover $U$ of $Y$ there exists a map $g: Y \rightarrow X$ such that $f \circ g$ is $U$-homotopic to $\operatorname{id}_{Y}$ and $g \circ f$ is $f^{-1}(U)$-homotopic to $\left.\operatorname{id}_{X}\right)$. Hence the Examples 4.2, 4.3 show that even one-to-one fine homotopy equivalences can raise dimension (cf. [A]). 


\section{REFERENCES}

[A] F. D. Ancel, Proper hereditary shape equivalences preserve property C, Topology and its Appl. (to appear).

[BP] C. Bessaga and A. Pelczynski, Selected topics in infinite-dimensional topology, PWN, Warsaw, 1975.

[H] W. E. Haver, Mappings between ANR's that are fine homotopy equivalences, Pacific J. Math. 58 (1975), 457-461.

[MM] J. van Mill and J. Mogilski, Property C and fine homotopy equivalences, Proc. Amer. Math. Soc. 90 (1984), 118-120.

Department of Mathematics, University of Tennessee, Knoxville, Tennessee 37916

Institute of Mathematics, University PKiN, 00 - 901 Warsaw, Poland (Current address of Jerzy Mogilski)

Current address (Mladen Bestvina): Department of Mathematics, University of California, Berkeley, California 94720 